\title{
Three-dimensional musculoskeletal modelling of the seated row resistance-training exercise
}

K Nolte, ${ }^{1} \mathrm{PhD} ; \mathbf{P}$ E Krüger, ${ }^{1} \mathrm{PhD} ; \mathbf{P}$ S Els, ${ }^{2} \mathrm{PhD} ; \mathbf{H}$ W Nolte, ${ }^{3} \mathrm{PhD}$

${ }^{1}$ Department of Biokinetics, Sport and Leisure Sciences, University of Pretoria, Pretoria, South Africa

${ }^{2}$ Department of Mechanical and Aeronautical Engineering, University of Pretoria, Pretoria, South Africa

${ }^{3}$ Ergonomics Technologies, Research and Development, Armscor, South Africa

Corresponding author: KNolte (kim.nolte@up.ac.za)

Objective. To evaluate whether three-dimensional (3D) musculoskeletal modelling could be effective in assessing the safety and efficacy of exercising on a seated row resistance-training machine. The focus of the evaluation was on biomechanical and anthropometric considerations of the end user.

Methods. Three anthropometric cases were created; these represented a 5th percentile female as well as a 50th and a 95th percentile male based on body mass index. Two repetitions, with a resistance equal to $50 \%$ of the functional strength of one repetition maximum (1RM) for each anthropometric case, were performed.

Results. Results indicate that the default model of the LifeModeler software has important limitations that should be taken into consideration when used to evaluate exercise equipment. Adjustments had to be made to the model to solve the forward dynamics simulations; as a result, no muscle forces or contraction values were obtained. This negatively influenced the value of the results as these parameters are important when analysing an exercise. The seated row resistance-training machine's engineered or manufactured adjustability was sufficient, as it appeared to accommodate the three anthropometric cases adequately during execution of this exercise. Conclusion. It appears that 3D musculoskeletal modelling can be used to evaluate resistance-training exercises such as the seated row; however, the limitations indicated by this study must be taken into consideration, especially when using the default LifeModeler model.

S Afr J SM 2013;25(3):67-73. DOI:10.7196/SAJSM.470

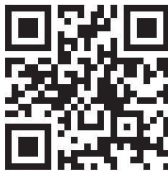

The advancement in computer technology and data processing capability has allowed the improvement of modelling software to a point where dynamic problems can now be simulated and analysed in a digital environment. ${ }^{[1]}$ With the capability to simulate musculoskeletal human models interacting with mechanical systems, many aspects concerning the effects of the resistance-training equipment on the body can be studied.

In recent years, the popularity of dynamic resistance training has risen. This type of training is suitable for developing muscular fitness 
of men and women of all ages, as well as of children. ${ }^{[2]}$ The seated row forms the basis of many land-based training programmes for athletes, more specifically rowers. However, it is also often included as part of strength-training programmes for non-athletes. It is an effective exercise to strengthen the musculature of the upper back. The primary joint movements of this exercise are shoulder extension and elbow flexion, thus the prime movers include the latissimus dorsi and the biceps brachii muscles. ${ }^{[2]}$ Other important muscles involved in the seated row exercise are the posterior deltoids, trapezius and rhomboideus muscle groups. ${ }^{[3]}$ In terms of understanding the biomechanics associated with various resistance-training exercises, a great deal of literature has investigated the kinetics and kinematics associated with the bench press, squat and Olympic lifts. Therefore, it would appear that there has been a preoccupation with extension-type tasks and very little attention has been given to other movements. ${ }^{[4]}$ Furthermore, much of the available research focuses on rowing ergometer analysis rather than the seated row resistance exercise.

Here we present the musculoskeletal modelling of three anthropometric cases upon exercising on a commercially available seated row resistance-training machine. The primary aim of this study was to determine the efficacy of three-dimensional (3D) musculoskeletal modelling in evaluating resistance-training equipment design such as the seated row resistance-training machine.

\section{Methods}

\section{Equipment}

A 3D full-body musculoskeletal model was created using LifeModeler software and incorporated into a multibody dynamics model of the seated row resistance machine generated in MSC ADAMS software (Fig. 1). LifeModeler runs as a plug-in of MSC ADAMS. It has previously been used in studies in the fields of sport, exercise and medicine. ${ }^{[5,6]}$ It was decided to evaluate a default model as generated by the software. This model consists of 19 segments including a base set of joints for each body region. Specifically, the spine does not consist of individual vertebrae, but rather of various segments that represent different regions of the vertebral column, with joints between these segments. Furthermore, the default model has a fullbody set of 118 muscle elements attached to the bones at anatomical landmarks, including most of the major muscle groups in the body. Closed-loop simple muscles were modelled. Closed-loop muscles contain proportional-integral-differential (PID) controllers. The PID controller algorithm uses a target length $\mathrm{v}$. time curve to generate the muscle activation and the muscles follow this curve. Because of this approach, an inverse dynamics simulation using passive recording muscles is required prior to simulation with closed-loop muscles. Simple muscles fire with no constraints, except for the physiological cross-sectional area (pCSA), which designates the maximum force that a muscle can exert. The graphs of simple muscle activation curves will generally peak at a flat-force ceiling value. ${ }^{[7]}$

\section{Musculoskeletal full-body human and seated row computer-aided design (CAD) models}

Three anthropometric cases were created for each piece of equipment. The human models were created using the GeBOD anthropometry database (default LifeModeler database), but were based on body mass index (BMI) data obtained from RSA-MIL-STD 127 Vol. $1^{[8]}-\mathrm{a}$ representative anthropometry standard of the South African National Defence Force (SANDF) that is kept current by a yearly sampling plan, and is an accurate representation of the broader South African (SA) population. Bredenkamp ${ }^{[9]}$ described a process to characterise the body forms of SANDF males and females. Body form variances described by two principle components (PCs) for the SANDF males and two PCs for SANDF females were included in the modelling process. Positive and negative boundary cases of each PC, representing the boundary conditions to be accommodated in design, identified the total range of four male and four female models. ${ }^{[10]}$ The first PC described the 'fatness' variance in the population. Anthropometric variables included for this PC are bust, waist and hip circumferences, together with BMI and bust-to-waist ratio. The second PC described the length variances in the population. Anthropometric variables included for this $\mathrm{PC}$ are stature, inside-arm length and crotch length. It was decided to use the cases representing the smallest female as well as an average and large male for the three anthropometric cases for this study. These cases could be seen as what are traditionally known as a 5th percentile female, 50th percentile male and a 95th percentile male based on the BMI of each of these cases. Thus, for the purpose of building these biomechanical models, a correlation between BMI and functional body strength was assumed. Similar assumptions have previously been made in biomechanics full-body model simulations. ${ }^{[11]}$ Annegarn et al. ${ }^{[12]}$ also verified scaled modelling strengths against actual functional body strengths and correlations ranged from 0.64 to 0.99 .

This approach was followed to ensure that the equipment could accommodate an acceptable sample of the SA end-user population. A CAD model of the seated row resistance-training machine was obtained from an exercise equipment manufacturing company in SA. The model in a Parasolid file format was imported into the LifeModeler simulation software.

The MSC ADAMS software was used to create two design variables in order to adjust the external resistance (as selected by the amount of weights when using a selectorised resistance-training machine) and to specify the radius of the cam over which the cable of an actual exercise machine would run in order to lift the selected resistance. This was possible since this machine employed a circular cam system. A special contact force (solid to solid) was created between the weights being lifted and the remainder of the weight stack during the simulation. A coupler joint was created, linking the revolute joint (driver) of the lever arm attached to the handle bars with the translational joint of the weight stack. The design variable created for the radius of the cam was referenced as the scale of the coupled joint (translational joint at weights). The design variable created for the mass of the weights was then adjusted according to the pre-determined resistance for each anthropometric case.

The external resistance applied in the models was based on data obtained from RSA-MIL-STD 127 Vol. 5. ${ }^{[13]}$ This database consists of a range of human functional-strength measurement variables for SANDF males and females. This standard can be considered an accurate representation of the functional body strength of the SA population. ${ }^{[13]}$ Furthermore, functional strength data were used from activities that most closely resembled the movements of the exercise as well as the muscle groups used during such movement. Fifty per cent of the functional strength of one repetition maximum (1RM) for each anthropometric case was used as this can be considered a 
manageable resistance to perform an exercise with appropriate form and technique for two repetitions. The end of the concentric phase of each repetition was when the upper arms reached the anatomical zero position (neutral) alongside the torso, after which the eccentric phase of each repetition returned the model to the starting position described in Table 1.

\section{Simulation}

Extreme care was taken with the positioning of the musculoskeletal model on the seated row machine to ensure that technique, posture and positioning were correct according to best exercise principles (Table 1). Optimal positioning of the models on the equipment required approximately $90^{\circ}$ of shoulder flexion with slight elbow flexion that resulted in the hands finally being just higher than the elbows for all anthropometric cases. This would be considered the correct posture for this exercise and resulted in the handle height being just below shoulder level for all cases. Furthermore, total manufacturer adjustability of the exercise machine was used in order to ensure correct positioning for each case. The following steps were performed to ensure realistic kinematics during the inverse dynamics simulations: (i) positioning of the human model on the exercise equipment; (ii) adjustment of the posture to allow for the human-machine interface to be created; (iii) creating the constraints between the human and machine; (iv) prescribing the motion of the repetitions; ( $v$ ) evaluation of the resultant kinematics; and ( $v i$ ) adjustment of joint positions until inverse dynamics resulted in a realistic exercise movement. Bushing elements were used to secure the chest to the chest pad/cushion as well as the lower torso to the seat, and spherical joints were used to connect the hands to the handlebars of the seated row machine. Bushing elements were preferred to fixedjoint elements; the former allows limited translational and rotational motion, and the amount of motion can be controlled by changing stiffness and damping characteristics in all three orthogonal directions. The original joints created in the biomechanical model had default joint parameters (stiffness $(\mathrm{K})=1 \mathrm{E} 4$, dampening $(\mathrm{C})=1000$ ). Joints with such high joint stiffness are created to ensure a relatively 'rigid' model that provides a stable and smooth motion when manipulated by motion splines. This is especially important during the movement of the model into the initial posture, and to ensure smooth model motion during inverse dynamics. After the muscle lengths had been recorded in the inverse dynamics, the joint stiffness was changed to near zero, to represent actual stiffness in normal and healthy human joints.

The inverse dynamics/forward dynamics method was applied during the simulations. Inverse dynamics simulations are performed on models that are being manipulated by the use of motion agents or motion spines. During the inverse dynamics simulation, a rotational motion was applied to the revolute joint of the lever arm attached to the handlebars of the seated row machine in order to generate the required movement of the resistance-training machine. This movement replicated the pulling (concentric) and resisting (eccentric) phase of the exercise. The time for

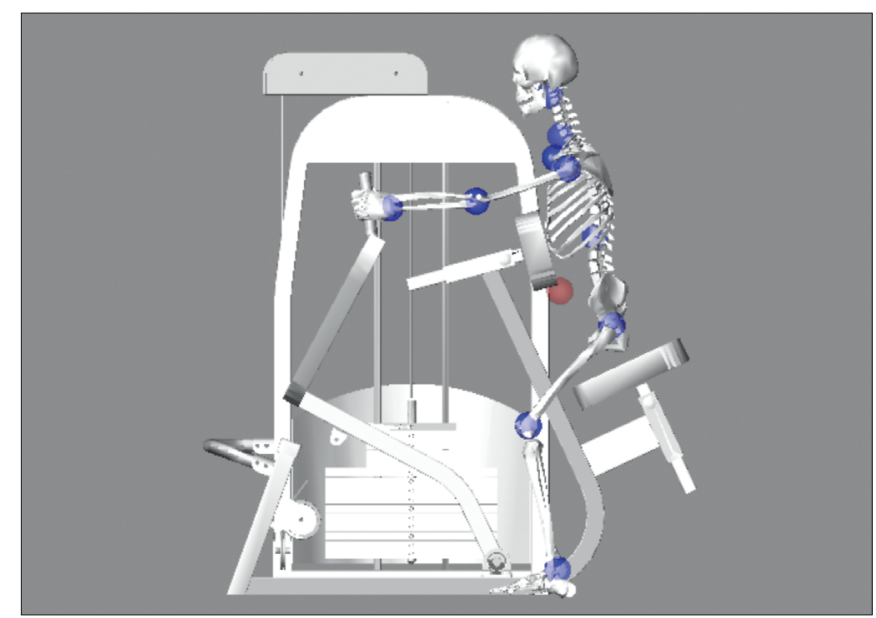

Fig. 1.3D musculoskeletal modelling of the seated row resistance-training machine and 50th percentile male musculoskeletal model using LifeModeler and MSC ADAMS software.

Table 1. Exercise starting posture for the three anthropometric cases on the seated row machine ${ }^{\star}$

\begin{tabular}{|c|c|c|c|}
\hline Joint & 5th percentile female & 50th percentile male & 95th percentile male \\
\hline Scapula $^{\dagger}$ & $0.0 ; 0.0 ; 0.0$ & $0.0 ; 0.0 ; 0.0$ & $0.0 ; 0.0 ; 0.0$ \\
\hline Shoulder ${ }^{\dagger}$ & $85.0(\mathrm{~F}) ; 5.0(\mathrm{IR}) ; 7.0(\mathrm{AB})$ & $85.0(\mathrm{~F}) ; 5.0(\mathrm{IR}) ; 4.5(\mathrm{AB})$ & $85.0(\mathrm{~F}) ; 5.0(\mathrm{IR}) ; 2.5(\mathrm{AB})$ \\
\hline Elbow $^{\dagger}$ & 15.0 (F); 10.0 (IR); 0.0 & 15.0 (F); 10.0 (IR); 0.0 & 15.0 (F); 10.0 (IR); 0.0 \\
\hline Wrist $^{\dagger}$ & $0.0 ; 0.0 ; 0.0$ & $0.0 ; 0.0 ; 0.0$ & $0.0 ; 0.0 ; 0.0$ \\
\hline $\mathrm{Hip}^{\dagger}$ & $30.0(\mathrm{~F}) ; 0.0 ; 0.0$ & $35.0(\mathrm{~F}) ; 0.0 ; 0.0$ & $52.0(\mathrm{~F}) ; 0.0 ; 0.0$ \\
\hline Knee $^{\dagger}$ & $30.0(\mathrm{~F}) ; 0.0 ; 0.0$ & $45.0(\mathrm{~F}) ; 0.0 ; 0.0$ & $60.0(\mathrm{~F}) ; 0.0 ; 0.0$ \\
\hline Ankle $^{\dagger}$ & $0.0 ; 0.0 ; 0.0$ & $12.0(\mathrm{E}) ; 0.0 ; 0.0$ & $12.0(\mathrm{E}) ; 0.0 ; 0.0$ \\
\hline Upper neck & $0.0 ; 0.0 ; .0 .0$ & $0.0 ; 0.0 ; 0.0$ & $0.0 ; 0.0 ; 0.0$ \\
\hline Lower neck & $0.0 ; 0.0 ; .0 .0$ & $0.0 ; 0.0 ; 0.0$ & $0.0 ; 0.0 ; 0.0$ \\
\hline Thoracic & $0.0 ; 0.0 ; .0 .0$ & $0.0 ; 0.0 ; 0.0$ & $0.0 ; 0.0 ; 0.0$ \\
\hline Lumbar & $15.0(\mathrm{~F}) ; 0.0 ; 0.0$ & $15.0(\mathrm{~F}) ; 0.0 ; 0.0$ & $15.0(\mathrm{~F}) ; 0.0 ; 0.0$ \\
\hline \multicolumn{4}{|c|}{$\mathrm{F}=$ flexion; $\mathrm{E}=$ extension; $\mathrm{IR}=$ internal rotation; $\mathrm{AB}=$ abduction. } \\
\hline \multicolumn{4}{|c|}{${ }^{*}$ Results are presented for the sagittal, transverse and frontal planes (degrees). } \\
\hline${ }^{+}$The joint angles re & & & \\
\hline
\end{tabular}


Table 2. User population anthropometric and strength data

\begin{tabular}{llll}
\hline User population group & Body mass $(\mathbf{k g})$ & Stature $(\mathbf{m m})$ & Exercise resistance $\mathbf{5 0 \%}$ of $\mathbf{1 R M}(\mathbf{k g})$ \\
\hline 5th percentile female & 49.5 & 1500 & 11 \\
50th percentile female & 66.0 & 1610 & 18 \\
95th percentile male & 85.0 & 1840 & 30
\end{tabular}

the concentric phase was set at $1.66 \mathrm{~s}$ and the eccentric phase longer at $3.33 \mathrm{~s}$ to mimic conventional resistance-training technique in which the eccentric phase is more deliberate to prohibit the use of momentum. The $1.66 \mathrm{~s}$ concentric phase included a STEP function approximation over $0.5 \mathrm{~s}$ to ensure a gradual start to the movement. In MSC ADAMS, the STEP function approximates an ideal mathematical step function. It steps quantities such as motions or forces up or down, or on and off. A STEP function is used when a value needs to be changed from one constant to another. The joints forces of the model were recorded during the inverse dynamics simulation in order to calculate the changes in joint torques to result in the required machine movement.

After the inverse dynamics simulation was performed, the rotational motion was removed from the rotational joint of the lever arm of the seated row machine. The resulting joint movements were then used to drive the model during the forward dynamics simulation in the manner as developed through the inverse dynamics simulation. During the forward dynamics simulation, the model is guided by the internal forces (muscle-length changes resulting in joint angulations and torques) and influenced by external forces (gravity, contact and determined exercise resistance). It is important to note that changes had to be made to the LifeModeler default model in order to solve the models with plausible kinematics during the forward dynamics simulations. Considering the research problem, the detail of these changes will be discussed under the discussion section. All results presented are derived from the forward dynamics simulations after these changes to the default model were made.

\section{Data analysis}

Firstly, we determined if the forward dynamics simulations could adequately be solved by the LifeModeler default model. Kinematic data obtained from the inverse dynamics simulations were visually compared with those of the forward dynamics simulations to determine if the data were plausible.

Secondly, the anthropometric dimensions and exercise postures of the musculoskeletal human models were visually assessed in relation to the dimensions and adjustability of the resistance-training equipment in order to determine if all three anthropometric cases representative of the SA end-user population could be accommodated comfortably on the seated row resistance-training machine. Key aspects included start and end exercise posture, as well as maintaining correct technique throughout the exercise during the simulations. Correct technique was assessed in terms of limited compensatory movements and performing the seated row exercise through the full range of motion as determined by the inverse dynamics.

Lastly, to determine exercise safety and efficacy, joint forces were evaluated. The risk of injury to the musculoskeletal system of the exerciser was ascertained by comparison of measured forces with safe loading limits for joints of the lumbar and thoracic spine. Risk to both these structures are real during exercises that require pulling or pushing movements (with and without resistance) and/or during the execution of exercises with poor postures. Different joint-loading criteria were derived using biomechanical research, taking into consideration the posture and anthropometry. ${ }^{[14]}$ However, criteria for determining whether a particular task or exercise is 'safe' based on tissue-level stresses or joint loading are available only for a small number of tissues and loading regimes (e.g. lower-back motion segments in compression): ${ }^{[1]}$ therefore, for this study, anterior/posterior (A/P) shear forces and joint compression forces were used as safety criteria.

Basic descriptive statistical analyses were completed with Satistica.

\section{Results}

Three anthropometric cases based on BMI data obtained from RSAMIL-STD 127 Vol. $1^{[13]}$ were used for the study, and results were assessed (Table 2). Table 2 represents the external resistance applied for each anthropometric case.

Due to the involvement of the wrist, elbow and shoulder joints in the seated row exercise, torque values for these joints are presented in Table 3. The 95th percentile male recorded the highest peak joint torque values for the three joints. The 50th percentile male's peak elbow and wrist torque values were the lowest. The peak shoulder torque values of the 5 th percentile female and 50th percentile male were similar and were lower than the 95th percentile male's values. For the three anthropometric cases, the peak shoulder joint torque values were the lowest, followed by the wrist and the greatest for the elbow.

The seated row exercise is a multi-joint exercise, thus movement in the sagittal plane of the shoulder, elbow and wrist (right side) are reported (Table 3). The least movement occurred at the wrist joint, followed by the shoulder joint, with the most movement at the elbow joint for the three anthropometric cases. Range of motion of the 5th percentile female was the least for the wrist and shoulder joints. Range of motion was the greatest for the 95th percentile male in the wrist and shoulder joint. Elbow joint range of motion was greatest for the 50th percentile male.

Results for the thoracic (T12/L1 intervertebral joint) and lumbar (L5/S1 intervertebral joint) spine compression and A/P shear forces are presented in Table 4. Peak thoracic spine joint compression forces were greatest for the 50th percentile male, followed by the 95th percentile male, and were lowest in the 5th percentile female. There was a similar trend in the peak lumbar spine joint compression forces. In all anthropometric cases, the peak lumbar spine joint compression forces were greater than the peak thoracic spine joint compression forces. The 5 th percentile female and 50th percentile male recorded similar peak thoracic and lumbar spine $\mathrm{A} / \mathrm{P}$ shear forces. The 5th percentile female's peak thoracic spine and lumbar $\mathrm{A} / \mathrm{P}$ shear forces were the least in comparison with the other two anthropometric cases. 
Table 3. Right wrist, elbow and shoulder joint torque (Nm) and joint angle $\left(^{\circ}\right)$ results in the sagittal plane for the three anthropometric cases ${ }^{*}$

\begin{tabular}{|c|c|c|c|}
\hline & Mean & Minimum & Maximum \\
\hline \multicolumn{4}{|l|}{ 5th percentile female } \\
\hline Wrist torque $(\mathrm{Nm})$ & -1.6 & -4.5 & 0.0 \\
\hline Wrist angle $\left({ }^{\circ}\right)$ & 16.0 & 0.0 & 26.5 \\
\hline Elbow torque $(\mathrm{Nm})$ & -4.0 & -6.3 & 0.0 \\
\hline Elbow angle $\left(^{\circ}\right)$ & -75.8 & -129.6 & -15 \\
\hline Shoulder torque (Nm) & 0.9 & -1.2 & 3.2 \\
\hline Shoulder angle $\left({ }^{\circ}\right)$ & -52.0 & -85.0 & -16.4 \\
\hline \multicolumn{4}{|l|}{ 50th percentile male } \\
\hline Wrist torque (Nm) & -1.3 & -3.1 & 0.0 \\
\hline Wrist angle $\left({ }^{\circ}\right)$ & 16.3 & 0.0 & 27.5 \\
\hline Elbow torque (Nm) & -3.0 & -4.7 & 0.0 \\
\hline Elbow angle $\left(^{\circ}\right)$ & -75.9 & -130.5 & 15.0 \\
\hline Shoulder torque (Nm) & 0.2 & -1.2 & 1.9 \\
\hline Shoulder angle $\left(^{\circ}\right)$ & -53.7 & -85 & -20.8 \\
\hline \multicolumn{4}{|l|}{ 95th percentile male } \\
\hline Wrist torque (Nm) & -0.2 & -4.8 & 2.3 \\
\hline Wrist angle $\left({ }^{\circ}\right)$ & 17.1 & 0.0 & 29.0 \\
\hline Elbow torque (Nm) & -13.3 & -19.5 & 0.0 \\
\hline Elbow angle $\left(^{\circ}\right)$ & -73.2 & -125.9 & -15.0 \\
\hline Shoulder torque (Nm) & 1.7 & -2.5 & 7.0 \\
\hline Shoulder angle $\left({ }^{\circ}\right)$ & -57.8 & -85.0 & -28.6 \\
\hline
\end{tabular}

The results for wrist and elbow-joint $\mathrm{A} / \mathrm{P}$ shear forces are presented in Table 4. Peak wrist and elbow-joint A/P shear forces were lowest for the 50th percentile male and highest for the 95th percentile male. Peak wrist A/P shear forces were slightly lower than elbow shear forces for all the anthropometric cases.

\section{Discussion}

Firstly, it can be concluded that the LifeModeler default model was not adequate to solve the forward dynamics simulations for any of the anthropometric cases. The same conclusion ${ }^{[15]}$ was drawn in a previous study that evaluated the seated biceps curl resistancetraining exercise. For the evaluation of the seated biceps curl exercise, the forward dynamics simulations could also only be solved after a number of adjustments had been made to the model, such as increasing the pCSA of the muscles, manipulating muscle origins and insertions, and decreasing the joint stiffness in the forwards dynamics simulations. ${ }^{[15]}$ All of these adjustments were implemented for this study in order to solve the simulation, without any success. Possible reasons for this include the degrees of freedom involved in a multijoint exercise involving highly mobile joints such as the shoulder. Furthermore, it could be that additional musculature is required
Table 4. Joint compression and $\mathrm{A} / \mathrm{P}$ shear forces $(\mathrm{N})$ for the three anthropometric cases

\begin{tabular}{|c|c|c|c|}
\hline & \multicolumn{3}{|c|}{$\mathbf{N}$} \\
\hline & Mean & Minimum & Maximum \\
\hline \multicolumn{4}{|l|}{5 th percentile female } \\
\hline \multicolumn{4}{|c|}{ Compression forces* } \\
\hline Thoracic spine & 100.3 & 79.4 & 149.1 \\
\hline Lumbar spine & 145.0 & 124.1 & 193.8 \\
\hline \multicolumn{4}{|l|}{ A/P shear forces ${ }^{\dagger}$} \\
\hline Thoracic spine & -22.0 & -30.8 & -18.3 \\
\hline Lumbar spine & -22.0 & -30.8 & -18.3 \\
\hline Wrist & 42.0 & 16.3 & 55.7 \\
\hline Elbow & 41.9 & 10.3 & 56.6 \\
\hline \multicolumn{4}{|l|}{ 50th percentile male } \\
\hline \multicolumn{4}{|c|}{ Compression forces* } \\
\hline Thoracic spine & 140.0 & 113.7 & 168.1 \\
\hline Lumbar spine & 200.0 & -173.2 & 227.6 \\
\hline \multicolumn{4}{|l|}{$\mathrm{A} / \mathrm{P}$ shear forces ${ }^{\dagger}$} \\
\hline Thoracic spine & -31.4 & -36.6 & -18.3 \\
\hline Lumbar spine & -31.4 & -36.6 & -18.3 \\
\hline Wrist & 29.7 & 16.6 & 42.8 \\
\hline Elbow & 29.7 & 10.2 & 43.8 \\
\hline \multicolumn{4}{|l|}{ 95th percentile male } \\
\hline \multicolumn{4}{|c|}{ Compression forces* } \\
\hline Thoracic spine & -32.7 & -97.1 & 162.8 \\
\hline Lumbar spine & 28.2 & -36.1 & 223.9 \\
\hline \multicolumn{4}{|l|}{ A/P shear forces ${ }^{\dagger}$} \\
\hline Thoracic spine & 0.4 & -36.2 & -12.4 \\
\hline Lumbar spine & 0.4 & -36.2 & -12.4 \\
\hline Wrist & 103.6 & 31.7 & 122.4 \\
\hline Elbow & 103.5 & 18.7 & 124.1 \\
\hline \multicolumn{4}{|c|}{$\mathrm{A} / \mathrm{P}=$ anterior$/$ posterior. } \\
\hline \multicolumn{4}{|c|}{$\begin{array}{l}\text { * Positive values indicate forces in a superior direction and negative values indicate forces in an } \\
\text { inferior direction. }\end{array}$} \\
\hline $\begin{array}{l}{ }^{\top} \text { Positive indicates forces in a } \\
\text { direction. }\end{array}$ & direction at & negative indicate $\mathrm{f}_{\mathrm{f}}$ & ces in an anterios \\
\hline
\end{tabular}

to provide more stability in the shoulder joint during the forward dynamics simulations. To solve this problem in this study, the joint angulations recordings in the inverse dynamics simulations were used to solve the forward dynamics simulations. While this approach should still result in plausible compression and $\mathrm{A} / \mathrm{P}$ shear reaction forces, the muscle-length changes resulting in such compression forces are not available for evaluation. Rather, this option creates a trained PID-servo-type controller on the joint axis. The joint is commanded to track an angular history spline with a user-specified gain on the error between the actual angle and the commanded error. A user-specified derivative gain is specified to control the derivative of the error. Therefore, results for muscle forces $(\mathrm{N})$ and contractions 
(shortening and lengthening) ( $\mathrm{mm}$ ) could not be analysed. Ideally these parameters should be analysed when evaluating an exercise. It appears that more complex, multi-joint or compound exercises that require too many degrees of freedom pose a problem for the default model; therefore, models with more detailed musculature may be required to solve the forward dynamics simulations sufficiently. Important musculature required for the performance of the seated row exercise that are not included in the LifeModeler default model are the rhomboideus major and minor and the rotator cuff group (supraspinatus, infraspinatus, teres minor and subscapularis). It was not, however, within the scope of this study to produce anatomical detailed models, but rather to evaluate the default model of the software.

Secondly, the study did not indicate any obvious discrepancies between the anthropometric dimensions of the three cases and the seated row machine's engineered or manufactured adjustability. All three anthropometric cases appeared to be positioned adequately on the seated row machine. This was not the case with previous studies conducted on the abdominal crunch and seated biceps curl machines, which demonstrated the inability of the machines to adjust appropriately to individuals with small anthropometric dimensions, such as some women and children. ${ }^{[15,16]}$ As a result the exercise technique of the 5th percentile female was negatively influenced and injury risk was increased for these two exercises. ${ }^{[15,16]}$

Lastly, with regards to the biomechanical evaluation in terms of exercise efficacy and injury risk, the following could be deduced from the study: due to the fact that the forward dynamics simulations was solved by recording the joint angulations changes during the inverse dynamics simulations and not muscle-length changes, results for the muscle forces and contractions were not obtained and could therefore not be analysed. This negatively influenced the value of modelling with regards to evaluating the seated row exercise, as muscle forces and contractions provide important information regarding the efficacy and injury risk of the exercise.

Maximal joint torque values obtained for the wrist, elbow and shoulder appear to be lower when comparing the values to peak values obtained by means of isokinetic testing at $60 \%$ s, e.g.: wrist flexion and extension values of $13.8 \mathrm{Nm}$ and $12.7 \mathrm{Nm}$, respectively, in nondisabled subjects; ${ }^{[17]}$ elbow flexion and extension values of $36 \mathrm{Nm}$ for both elbow flexion and extension in female college basketball players; and shoulder flexion and extension values of $77 \mathrm{Nm}$ and $53 \mathrm{Nm}$ for males and $38 \mathrm{Nm}$ and $24 \mathrm{Nm}$ for females, respectively, in a group of non-disabled. ${ }^{[18,19]}$ Joint torque values for the three joints evaluated were much lower than values obtained during peak isokinetic testing; however, it is important to bear in mind that the values obtained in this study were not obtained from maximal testing as with the isokinetic testing. The peak elbow joint torque was the highest recorded value for all joints in the three anthropometric cases, which was too be expected as the elbow joint is most involved in the seated row movement.

Not surprisingly, the joint range of motion (wrist, elbow and shoulder) used during the seated row exercise was smallest for the 5th percentile female and greatest for the 95th percentile male; with the exception of the elbow joint range of motion, which was greatest for the 50th percentile male. It is not only important that correct technique is used for resistance exercises such as the seated row in order to decrease the likelihood of injury, but also that exercises are performed through the full range of motion to get the maximum benefits of the exercise.

In addition to lifting, pushing and pulling may also be associated with significant risk to the low back. ${ }^{[20]}$ The seated row exercise can be considered a pulling activity. It must be kept in mind that the cited research is primarily referring to occupational tasks; however, important similarities and conclusions can be drawn with exercises that use similar actions to occupational tasks and activities that require pulling. Furthermore, the spine of the default model does not consist of all the individual vertebrae, but rather of various segments that represent the different regions of the vertebral column with joints between these segments. Individualised vertebrae and corresponding joints may produce different results.

In 2009, a study by Knapik and Marras ${ }^{[21]}$ found that there was greater compressive loading at all spine levels when performing pulling compared with pushing activities. Therefore, an individual performing a pulling exercise such as the seated row may be at greater risk of a back injury than individuals performing a pushing exercise such as bench press, specifically with regards to compressive loading. ${ }^{[21]}$

Previous research from the American National Institute for Occupational Safety and Health $(\mathrm{NIOSH})^{[20]}$ recommended that spinal compression forces should not exceed $3.4 \mathrm{kN}$, to avoid injury. However, there is a very real threat of musculoskeletal injury before this failure limit value has been reached. ${ }^{[14,21]}$ British standards (BS EN 1005-3, 2002) recommend $600 \mathrm{~N}$ as the cut-off point for carrying masses; no further recommendations are made, except 'time of exposure needs to be minimised' and 'a preferred system requires optimal ergonomic position with reduced back bending posture. ${ }^{[22]}$ Therefore, all three anthropometric cases were well below the recommended failure limit of $3.4 \mathrm{kN}$. None of the anthropometric cases' peak thoracic or lumbar compression forces were even near the recommended $600 \mathrm{~N}$ cut-off; therefore, it may be postulated, all things considered, that the seated row exercise does not appear to cause excessive spinal compression forces that may put the individual at risk for an injury.

Historically, spine compression in the lower lumbar spine has been the variable of interest for risk to the low back during work and exercise training. However, during horizontal force application (pulling of the seated row exercise), it is expected that shear forces within the spine increase dramatically due to the application of force in the hands and the reaction of the trunk musculature. Thus, shear forces may represent the critical measure of risk. ${ }^{[21]}$ According to Knapik and Marras, ${ }^{[21]}$ in general, pushing activities impose greater, potentially risky A/P shear forces upon the spine than pulling. Pushing imposed up to $23 \%$ greater $\mathrm{A} / \mathrm{P}$ shear forces compared with pulling. Increases in shear forces were as a result of the increased flexor muscle co-activity required for the activity. ${ }^{[2]}$

Although the spine A/P shear forces recorded were greater than the compression forces, the thoracic and lumbar spine joint $\mathrm{A} / \mathrm{P}$ shear forces for the three anthropometric cases were also below the most commonly cited spine tolerance of $1000 \mathrm{~N}$ for shear force, as stipulated by McGill. ${ }^{[23]}$ It is important to note that even if the spine compression and $\mathrm{A} / \mathrm{P}$ shear forces recorded were well within acceptable limits, the modelling does not take into account the repetitive nature and accumulative effect of exercise. Further, the resistance used was only $50 \%$ of each case's estimated 1RM; therefore, if exercises use a resistance closer to their maximum, the loading values may exceed the acceptable limits. 
Handle height appears to affect the mechanical load of the low back and shoulder considerably, and it is recommended that carts are designed and are adjustable so that it is possible to push or pull at shoulder height. ${ }^{[24]}$ The same principle can be applied to the seated row machine: the handle bars should be approximately at shoulder height, which was the case for the three anthropometric models. Thus, this could have assisted in reducing the spine loads, especially the $\mathrm{A} / \mathrm{P}$ shear spine forces. Unfortunately, after conducting a literature search, it became clear that information regarding $\mathrm{A} / \mathrm{P}$ shear forces of the shoulder, elbow and wrist joints is scarce. However, the following information regarding handle height may be applicable in terms of reducing $\mathrm{A} / \mathrm{P}$ shear forces on these joints during the seated row exercise. Handle height and the magnitude of force are found to be significantly related to the net moment at the shoulder. Net moments at the shoulder are kept low during pushing and pulling activities by keeping the wrist, elbow and shoulder close to the line of action of the exerted force, or by directing the exerted force such that the shoulder joint remains close to the line of action of the exerted force. ${ }^{[24]}$ Thus, if the handle bars of the seated row resistance-training machine are designed in such a way as to ensure correct alignment of the shoulder, elbow and wrist joints, it may assist in reducing the strain that these joints experience during this exercise, especially if a heavy resistance is used.

\section{Conclusion}

Three-dimensional musculoskeletal modelling has value in the evaluation of the safety and efficacy of resistance-training equipment. This modelling method is a valuable tool for equipment design and may be of use to assess injury risk. However, musculoskeletal modelling that makes use of default models that lack adequate biofidelity does have limitations, as highlighted here. Adjustments had to be made to the default model to solve the forward dynamics simulations using recorded joint angulations during the inverse dynamics simulations. As a result, no muscle (force and contraction) results could be obtained which negatively affected the value of the modelling effort in evaluating the seated row exercise in terms of efficacy and safety. From an equipment design perspective, the anthropometric dimensions of the end-users appeared to be accommodated adequately by the seated row's engineered or manufactured adjustability. Practically, this study highlights the possible risk for spinal injury associated with pulling activities. Our results emphasise the importance of exercising with correct positioning and technique at an appropriate external resistance, to avoid undue strain on spinal structures.

\section{References}

1. Wagner D, Rasmussen J, Reed M. Assessing the Importance of Motion Dynamics for Ergonomic Analysis of Manual Materials Handling Tasks using the AnyBody
Modelling System. Proceedings of the 2007 Digital Human Modelling for Design and Engineering Conference, Seattle, Washington, 2007.

2. Heyward VH. Advanced Fitness Assessment and Exercise Prescription. 5th ed. Champaign, USA: Human Kinetics, 2004

3. Floyd RT. Manual of Structural Kinesiology. 17th ed. New York: McGraw-Hill, 2009

4. Cronin JB, Jones JV, Hagstrom JT. Kinematics and kinetics of the seated row and implications for conditioning. J Strength Cond Res 2007;21(4):1265-1270. [http:// dx.doi.org/10.1519/R-21246.1]

5. De Jongh C. Critical evaluation of predictive modelling of a cervical disc design Unpublished dissertation. Stellenbosch: University of Stellenbosch, 2007.

6. Hofmann M, Danhard M, Betzler N, et al. Modelling with BRG.lifeMODTM in sport science. Int J Comp Sci Sport 2006;5:68-71.

7. Biomechanics Research Group, Inc. LifeMOD Biomechanics Modeler Manual. San Clemente, USA: Life Modeler, 2006.

8. RSA-MIL-STD-127. Ergonomic design: Anthropometry and environment. Pretoria: RMSS, 2004:1-196.

9. Bredenkamp K. The Characterisation of the Male and Female Body Forms of the SANDF. Pretoria: ERGOTECH, 2007.

10. Gordon CC, Brantley JD. Statistical modelling of population variation in the head and face. Proceedings of the 1997 Design and Integration of Helmet Systems International Symposium, Massachusetts, USA, 1997.

11. Rasmussen J, de Zee M, Damsgaard A, et al. A General Method for Scaling Musculoskeletal Models. Paper presented at the International Symposium on Computer Simulation in Biomechanics, Cleveland, Ohio, USA, 2005.

12. Annegarn J, Rasmussen J, Savelberg HHCM, et al. Strength Scaling in Human Musculoskeletal Models. Paper presented at the European Workshop on Movement Sciences, Amsterdam, The Netherlands, 2007.

13. RSA-MIL-STD-127. Ergonomic Design: Biomechanics - Specific Functional Body Strength Data Standard. Pretoria: RMSS, 2001:1-28.

14. Cooper G, Ghassemieh E. Risk assessment of patient handling with ambulance stretcher systems (ramp/winch), easy-loader, tail-lift using biomechanical failure criteria. Med Eng Phys 2007;29:775-787. [http://dx.doi.org/10.1016/j.medengphy.2006.08.008]

15. Nolte K, Krüger PE, Els PS. Three dimensional musculoskeletal modelling of the seated biceps curl resistance training exercise. Sport Biomech 2011;10:146-160 [http://dx.doi.org/10.1080/14763141.2011.577441]

16. Nolte K, Krüger PE, Els PS, Nolte H. Three dimensional musculoskeletal modelling of the abdominal crunch resistance training exercise. J Sports Sci 2013;31:265-275. [http://dx.doi.org/10.1080/14763141.2011.577441]

17. Van Swearingen JM. Measuring wrist muscle strength. J Orthop Sports Phys Ther 1983;4:217-228.

18. Berg K, Blank D, Muller M. Muscular fitness profile of female college basketball players. J Orthop Sports Phys Ther 1985;7:59-64.

19. Nicholas JJ, Robinson LR, Logan A, et al. Isokinetic testing in young non-athletic able-bodied subjects. Arch Phys Med Rehabil 1989;70:210-213.

20. National Institute for Occupational Safety and Health. Musculoskeletal Disorder and Workplace Factors: A Critical Review of Epidemiologic Evidence for WorkRelated Musculoskeletal Disorders of the Neck, Upper Extremity, and Low Back. US Department of Health and Human Services (DHHS) Public Health Service, Centres for Disease Control. Cincinnati: National Institute for Occupational Safety and Health Division of Biomedical of Behavioural Science, 1997.

21. Knapik GG, Marras WS. Spine loading at different lumbar levels during pushing and pulling. Ergonomics 2009; 52(1):60-70. [http://dx.doi. org/10.1080/00140130802480828]

22. British Standards Institute. BS EN 1005 - 3. Safety of machinery - Human physical performance - Part 3: Recommended force limits for machinery operations. London: British Standards Institute, 2002

23. McGill SM. Searching for the safe biomechanical envelope for maintaining healthy tissue. Pre-Meeting workshop, International Society for the Study of the Lumbar Spine: The Contribution of Biomechanics to the Prevention and Treatment of Low Back Pain, University of Vermont, Burlington, 1996.

24. Hoozemans MJM, Kuijer PFM, Kingma I, et al. Mechanical loading of the low back and shoulders during pushing and pulling activities. Ergonomics 2004;47(1):1-18. [http://dx.doi.org/10.1080/00140130310001593577] 